\title{
Liver fibrosis staging with combination of APRI and FIB-4 scoring systems in chronic hepatitis $C$ as an alternative to transient elastography
}

\author{
Nikolaos Papadopoulos ${ }^{a}$, Sofia Vasileiadib, Maria Papavdib, Eirini Sveronia ${ }^{\text {, Pinelopi Antonakaki }}{ }^{\mathrm{b}}$, \\ Erminia Dellaporta ${ }^{b}$, Evangelia Koutli ${ }^{b}$, Stavroula Michalea ${ }^{b}$, Spilios Manolakopoulos ${ }^{b, c}$, John Koskinas ${ }^{b}$, \\ Melanie Deutsch ${ }^{b}$
}

417 Army Share Fund Hospital of Athens; $2^{\text {nd }}$ Academic Department of Internal Medicine, National and Kapodistrian University of Athens; Medical School of National and Kapodistrian University of Athens, Greece

Abstract

${ }^{a} 1^{\text {st }}$ Department of Internal Medicine, 417 Army Share Fund Hospital of Athens (Nikolaos Papadopoulos, Eirini Sveroni); ${ }^{\text {HHippokration }}$ General Hospital, Athens, Greece, $2^{\text {nd }}$ Academic Department of Internal Medicine, National and Kapodistrian University of Athens (Sofia Vasileiadi, Maria Papavdi, Pinelopi Antonakaki, Erminia Dellaporta, Evangelia Koutli, Stavroula Michalea, Spilios Manolakopoulos, John Koskinas, Melanie Deutsch); 'Laiko General Hospital, Athens, Greece, Department of Gastroenterology, Medical School of National and Kapodistrian University of Athens (Spilios Manolakopoulos), Greece

Conflict of Interest: None

Correspondence to: Nikolaos Papadopoulos, MD, PhD, Monis Petraki 10-12, 11521 Athens Greece e-mail: nipapmed@gmail.com

Received 6 May 2019; accepted 1 July 2019; published online 22 July 2019

DOI: https://doi.org/10.20524/aog.2019.0406

\section{Introduction}

Chronic infection with hepatitis $\mathrm{C}$ virus $(\mathrm{HCV})$ is a major and growing global public health problem, with more than 71.1 million persons infected worldwide [1]. In the era of new direct acting antivirals (DAAs), more than $95 \%$ of patients with chronic hepatitis $\mathrm{C}(\mathrm{CHC})$ can now be cured [2]. According to the recommendations of the European Association for the Study of the Liver for the treatment of hepatitis C in 2018, all patients with $\mathrm{CHC}$ willing to be treated, and who have no contraindications for treatment, should be treated. Moreover, it is recommended that patients with significant fibrosis (METAVIR F2/F3) or cirrhosis (F4) should be treated without delay [3].

However, an evaluation of liver disease severity is necessary prior to therapy. Patients with cirrhosis or advanced fibrosis 
(F3) are of particular importance, as the choice of treatment regimen and the post-treatment prognosis depend on the stage of fibrosis [3]. Liver biopsy has long been considered the gold standard for the assessment of liver fibrosis, but nowadays it has been almost entirely replaced by noninvasive methods that measure liver stiffness (LS), such as transient elastography (TE), or biochemical markers and scoring systems, such as the APRI score and FIB-4 scores [4,5]. The main advantage of biochemical noninvasive scores in evaluating liver fibrosis is that they are generally available at a low cost and are very simple to use. However, TE-LS measurement is not widely available, largely because of technical and practical reasons together with its high cost, while APRI and FIB-4 scores have been proved quite reliable for assessing liver fibrosis. However, validation in different patient populations is still needed [6,7].

In this study, the diagnostic performance of APRI and FIB4 scores compared to TE-LS in detecting F3/F4 patients was evaluated in a Greek CHC cohort. In addition, we estimated the best cutoff for these scores to predict the likelihood of advanced fibrosis or cirrhosis, in order to reduce the need for TE-LS.

\section{Patients and methods}

We reviewed the records of all patients with $\mathrm{CHC}$ who sought care and underwent TE-LS evaluation in 2 tertiary liver centers of Athens between May 2014 and August 2018. All patients were evaluated with a Fibroscan (Echosens, Paris, France) in the supine position, with the tip of the transducer placed on the skin between the ribs over the right lobe of the liver. Only the M probe was used for LS measurement, and at least 10 validated measurements with an interquartile range of $\leq 30 \%$ and a success rate of $>60 \%$ were required for a valid interpretation. While the correlation of TE-LS with METAVIR fibrosis stages has been established by several studies, different cutoffs for each stage of fibrosis have been proposed [6,8]. In our study the following stages of fibrosis were defined: F0 $1:<7$, F2: 7-8.9, F3: 9-11.9 and F4: $\geq 12 \mathrm{kPa}$, as these cutoff values have been adopted by the Greek National Insurance Program [6].

Patients who had coinfection with hepatitis B virus and/or human immunodeficiency virus, invalid TE-LS assessments and laboratory findings of acute hepatitis (alanine aminotransferase [ALT] levels $\geq 10$ upper limit of normal [ULN]) were excluded. The database included patient demographic and epidemiological characteristics, medical history, and clinical and laboratory data. The study was performed in accordance with the World Medical Association Declaration of Helsinki and was approved by the hospital's ethics committee.

\section{Laboratory methods}

Hematological and biochemical parameters were determined using commercially available assays. The upper limit of normal for both ALT and aspartate aminotransferase (AST) was $40 \mathrm{IU} / \mathrm{L}$. For each patient, the APRI score was calculated using the proposed formula: APRI $=[($ AST level/ULN $) /$ platelet count $\left.\left(10^{9} / \mathrm{L}\right)\right] \times 100$. The FIB-4 score was determined using the following formula: FIB- $4=$ [age $\times$ AST/platelet count $\left(10^{9} / \mathrm{L}\right) \times \sqrt{ }$ ALT $][9,10]$.

\section{Statistical analysis}

All data were analyzed using the statistical package MedCalc, version 18.11. Continuous variables are represented by their median (min-max) or mean \pm standard deviation. Statistical analysis was performed using the $t$-test for comparisons of continuous variables between groups and the corrected chisquared test for comparisons of qualitative data. Correlation analysis was used to compare the degree of hepatic fibrosis between TE-LS and both APRI and FIB-4 scores. Diagnostic performances for APRI and FIB-4 scores versus TE-LS were analyzed separately, according to sensitivity (Se), specificity $(\mathrm{Sp})$, negative predictive value (NPV), positive predictive value $(\mathrm{PPV})$ and area under the receiver operating characteristic (ROC) curve. The optimal cutoff value for each test was determined by applying the Youden index criterion. A twotailed P-value $<0.05$ was considered to be statistically significant.

\section{Results}

In total, 575 patients with $\mathrm{CHC}$ were evaluated. Their mean age was $51.54 \pm 12.4$ years and $63.5 \%$ of them were male. The vast majority (94\%) were Caucasians. The most frequent possible sources of infection were parenteral drug use in 269 (47\%), transfusion of blood or blood products before 1992 in 127 (22\%), and unknown in the remaining 179 (31\%). HCV genotype testing was performed in 551 (96\%) patients. Genotype $1 \mathrm{a}$ was detected in $20 \%$, genotype $1 \mathrm{~b}$ in $20 \%$, genotype 2 in $5 \%$, genotype 3 in $38.5 \%$ and genotype 4 in $16.5 \%$. The mean TE-LS score was $13.4 \pm 8.5 \mathrm{kPa}$, the median APRI score was $0.68(0.1-11.6)$ and the median FIB-4 score was 1.8 (0.2-19.4). Stages of liver fibrosis according to TE-LS were evaluated as F0-1 in $60(10.5 \%), \mathrm{F} 2$ in $150(26 \%), \mathrm{F} 3$ in $100(17.5 \%)$, and F4 in 265 (46\%) patients (Table 1).

Correlation analysis showed a significant positive correlation between TE-LS fibrosis stage and APRI ( $r=0.4515$, 95\% confidence interval $[\mathrm{CI}] 0.3839-0.5142 ; \mathrm{P}<0.0001)$ and FIB-4 ( $r=0.5374$, 95\%CI 0.4766-0.5930; $\mathrm{P}<0.0001)$.

Based on TE-LS fibrosis stage classification we estimated the optimal APRI and FIB-4 scores to predict the presence of liver cirrhosis (F4) and advanced fibrosis/cirrhosis (F3/F4) using ROC curve analysis (Table 2).

The optimal APRI score for F4 patients was calculated as $>0.65$ (area under the curve [AUC] 0.871, 95\%CI 0.84-0.897; $\mathrm{P}<0.0001$ ), giving Se $85.5 \%$, Sp 77\%, PPV 76\%, and NPV 86\% (Fig. 1). The optimal FIB-4 score for F4 patients was calculated as $>1.63$ (AUC 0.916, 95\%CI 0.89-0.937; $\mathrm{P}<0.0001$ ), giving Se 91\%, Sp 77\%, PPV 77\%, and NPV 91\% (Fig. 2). 
The optimal APRI score to predict F3/F4 patients as one group indicating advanced fibrosis or cirrhosis was calculated as $>0.64$ (AUC 0.82, 95\%CI 0.785-0.85; $\mathrm{P}<0.0001$ ), giving Se $72 \%$, Sp 83\%, PPV 88\%, and NPV 63\% (Fig. 3). The optimal FIB-4 score to predict $\mathrm{F} 3 / \mathrm{F} 4$ patients as one group was calculated as $>1.46$ (AUC 0.854, 95\%CI 0.822-0.882; $\mathrm{P}<0.0001$ ), giving $\mathrm{Se}$ 81.5\%, Sp 79\%, PPV 85.5\%, and NPV 71\% (Fig. 4).

The combination of optimal values $(>0.65$ and $>1.63$, respectively) for APRI and FIB-4 (APRI-FIB-4-COMBO) as one score predicted F4 patients with Se $82 \%$, Sp 89.5\%, PPV $87 \%$, and NPV 85\% $(r=0.7164, \mathrm{P}<0.0001)$. Furthermore, this combination with optimal values $>0.64$ and $>1.46$, respectively,

Table 1 Baseline characteristics of the 575 patients

\begin{tabular}{|c|c|}
\hline Characteristics & Value \\
\hline Male sex, n (\%) & $365(63.5)$ \\
\hline Age, years & $51.54 \pm 12.4$ \\
\hline Caucasian descent, n (\%) & $539(94)$ \\
\hline $\begin{array}{l}\text { Source of Infection, n (\%) } \\
\text { Parenteral drug use } \\
\text { Transfusion } \\
\text { Unknown }\end{array}$ & $\begin{array}{l}269(47) \\
127(22) \\
179(31)\end{array}$ \\
\hline ALT, IU/L & $71.8 \pm 62$ \\
\hline AST, IU/L & $62.3 \pm 48$ \\
\hline $\mathrm{PLT}, \mathrm{k} / \mu \mathrm{L}$ & $195 \pm 82$ \\
\hline $\begin{array}{l}\text { HCV genotypes, n/N (\%) } \\
\text { la } \\
1 \mathrm{~b} \\
2 \\
3 \\
4\end{array}$ & $\begin{array}{c}110 / 551(20 \%) \\
110 / 551(20 \%) \\
29 / 551(5 \%) \\
211 / 551(38.5 \%) \\
91 / 551(16.5 \%)\end{array}$ \\
\hline TE-LS, kPa & $13.4 \pm 8.5$ \\
\hline $\begin{array}{l}\text { Stages of liver fibrosis acco } \\
\text { TE-LS, } \mathrm{n} / \mathrm{N}(\%) \\
\text { F0-1 }(<7 \mathrm{kPa}) \\
\text { F2 }(7-8.9 \mathrm{kPa}) \\
\text { F3 }(9-11.9 \mathrm{kPa}) \\
\text { F4 }(\geq 2 \mathrm{kPa})\end{array}$ & $\begin{array}{c}60 / 575(10.5) \\
150 / 575(26) \\
100 / 575(17.5) \\
265 / 575(46)\end{array}$ \\
\hline APRI score & $0.68(0.1-11.6)$ \\
\hline FIB-4 score & $1.8(0.2-19.4)$ \\
\hline
\end{tabular}

TE, transient elastography; LS, liver stiffness predicted F3/F4 patients with Se 67\%, Sp 91.5\%, PPV 93\% and NPV 61.5\% $(r=0.566, \mathrm{P}<0.0001)$; hence, its use would avoid the need for TE assessment in $45.5 \%$ of referred patients. Thus,

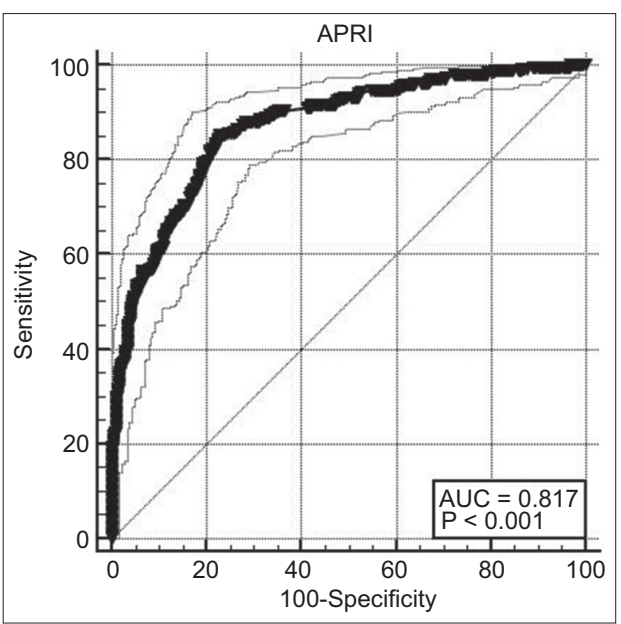

Figure 1 Performance of APRI score in the prediction of F4 patients according to TE-LS

TE, transient elastography; LS, liver stiffness; AUC, area under the curve

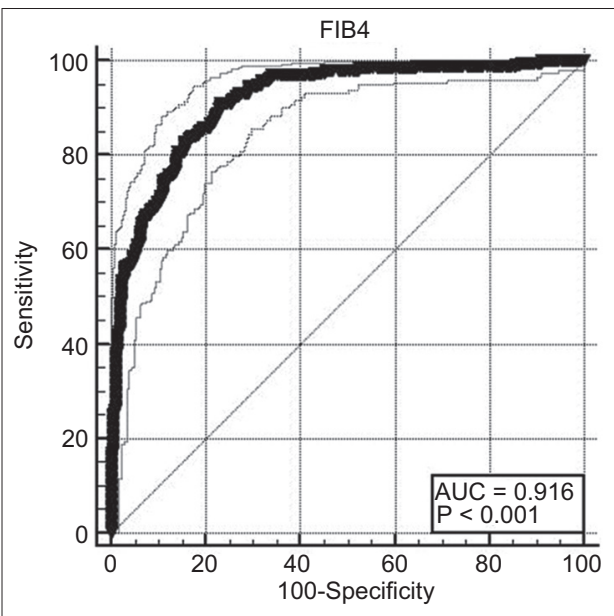

Figure 2 Performance of FIB-4 score in the prediction of F4 patients according to TE-LS

$T E$, transient elastography; LS, liver stiffness; AUC, area under the curve

Table 2 Performance indicators of APRI, FIB-4 and APRI/FIB-4 combination scores in F3/F4 and F4 patients

\begin{tabular}{|c|c|c|c|c|c|c|}
\hline \multirow{2}{*}{$\begin{array}{l}\text { Score } \\
\text { Fibrosis stage }\end{array}$} & \multicolumn{2}{|c|}{ APRI } & \multicolumn{2}{|c|}{ FIB-4 } & \multicolumn{2}{|c|}{ APRI-FIB-4-COMBO } \\
\hline & $\mathrm{F} 3 / \mathrm{F} 4$ & $\mathrm{~F} 4$ & $\mathrm{~F} 3 / \mathrm{F} 4$ & $\mathrm{~F} 4$ & $\mathrm{~F} 3 / \mathrm{F} 4$ & $\mathrm{~F} 4$ \\
\hline Cutoff value & $>0.64$ & $>0.65$ & $>1.46$ & $>1.63$ & $>0.64 />1.46$ & $>0.65 />1.63$ \\
\hline Sensitivity, \% & 72 & 85.5 & 81.5 & 91 & 67 & 82 \\
\hline Specificity, \% & 83 & 77 & 79 & 77 & 91.5 & 89.5 \\
\hline PPV, \% & 88 & 76 & 85.5 & 77 & 93 & 87 \\
\hline NPV, \% & 63 & 86 & 71 & 91 & 61.5 & 85.5 \\
\hline
\end{tabular}

$\overline{P P V}$, positive predictive value; $N P V$, negative predictive value 


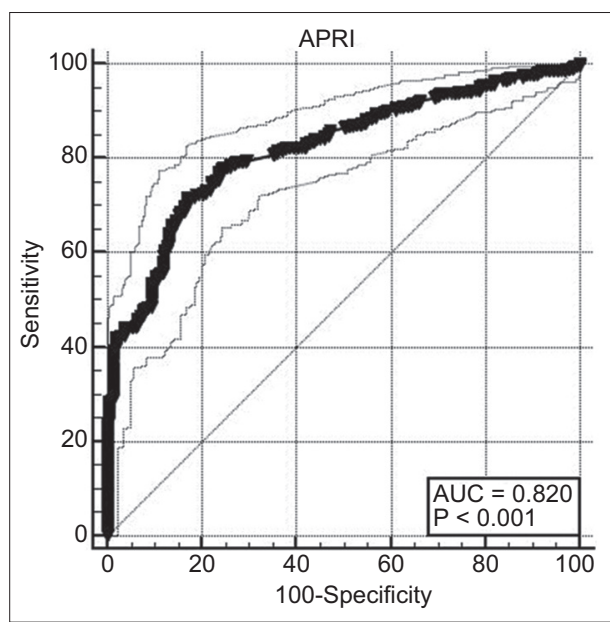

Figure 3 Performance of APRI score in the prediction of F3/F4 patients $A U C$, area under the curve

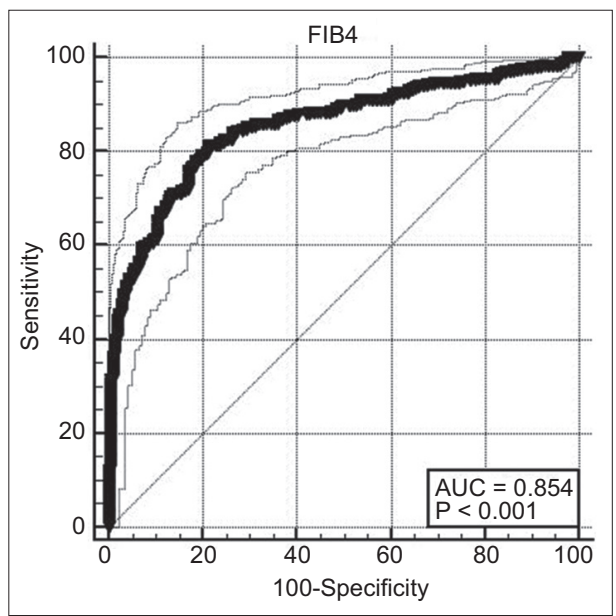

Figure 4 Performance of FIB- 4 score in the prediction of $\mathrm{F} 3 / \mathrm{F} 4$ patients $A U C$, area under the curve

it seems that the FIB- 4 score is adequate for ruling out non cirrhotic patients, while the APRI/FIB-4 combination has the best predictive ability in determining patients with advanced fibrosis or cirrhosis (F3/F4).

In order to achieve the maximum number of patients who may avoid the need of TE assessment, we performed an additional analysis, trying to define patients with an obviously low fibrosis score. In this context, using a lower cutoff APRI score of 0.3 or less would increase the Sp to $92.5 \%$, but reduce the Se to $34 \%$ for F1/F2 patients and would avoid the need for $\mathrm{TE}$ assessment in $17.5 \%$ of referred patients. Using a lower cutoff FIB-4 score of 0.98 or less would increase the Sp to $91.5 \%$, but reduce the Se to $40.5 \%$ for F1/F2 patients and would avoid the need for TE assessment in $20.5 \%$ of the referred patients. The APRI-FIB-4-COMBO score using these lower cutoff values ( 0.3 and 0.98 , respectively) predicted F1/ F2 patients with a Sp of $94.5 \%$ and a Se of $26.5 \%$ and would avoid the need for TE assessment in $13 \%$ of referred patients (Table 3).
Table 3 Lower APRI, FIB-4 and APRI/FIB-4 combination scores in the detection of patients with low fibrosis (F1/F2)

\begin{tabular}{lccc}
\hline Score & APRI & FIB-4 & APRI-FIB-4-COMBO \\
\hline Cutoff value & $\leq 0.3$ & $\leq 0.98$ & $\leq 03 / \leq 0.98$ \\
Sensitivity, \% & 34 & 40.5 & 26.5 \\
Specificity, \% & 92.5 & 91.5 & 94.5 \\
PPV, \% & 71.5 & 72.5 & 74 \\
NPV, \% & 71 & 73 & 69 \\
\hline PPV, positive predictive value; NPV, negative predictive value &
\end{tabular}

\section{Discussion}

Nowadays liver biopsy tends to be replaced by noninvasive tests. TE-LS is one of the most reliable methods for the assessment of liver fibrosis in CHC, but because of its high cost its use is not widespread in low- and middle-income countries [4]. On the other hand, APRI and FIB- 4 scores are 2 of the most popular scoring systems for liver fibrosis and have been reported to achieve high accuracy for the diagnosis of advanced fibrosis and cirrhosis in CHC patients compared to liver biopsy $[11,12]$. However, there is a lack of data comparing the diagnostic accuracy of both APRI and FIB-4 scores with that of TE-LS in $\mathrm{CHC}$ patients. In everyday practice, and taking into account the DAAs used today in most countries, the only information we need about the fibrosis stage is whether the patient has baseline advanced fibrosis or cirrhosis. This fact dictates the treatment duration ( 8 or 12 weeks), but is also extremely crucial in defining patients who need further evaluation, even after a successful sustained viral response (SVR), especially with regard to surveillance for hepatocellular carcinoma (HCC).

In the present study, we found that both scores predicted F4 (cirrhotic) patients adequately. The AUROC was 0.871 and 0.916 for the APRI and FIB-4 score, respectively. A threshold of $>0.65$ for APRI score was $85.5 \%$ sensitive and $77 \%$ specific in detecting F4 patients. The PPV of this threshold was as high as $76 \%$, with an NPV of $86 \%$. A threshold of $>1.63$ for FIB- 4 score was $91 \%$ sensitive and $77 \%$ specific in the diagnosis of F4 patients. The PPV of this threshold was $77 \%$ and the NPV was $91 \%$, indicating that FIB-4 is a satisfactory tool for ruling out non-cirrhotic patients.

However, while simply classifying $\mathrm{CHC}$ patients into cirrhotics and non-cirrhotics could be considered correct in order to decide the duration of the DAA treatment, it is insufficient to distinguish patients who need post-SVR HCC surveillance from those who do not. For this purpose an accurate tool for discriminating between the cohorts of F3 and F4 patients (advanced fibrosis and cirrhosis) is needed.

It is well known that any noninvasive biochemical markers and scores are less effective per se at defining intermediate degrees of fibrosis [13]. Thus, we conducted an analysis of F3 and F4 patients as one group, indicating significant fibrosis, in an attempt to define an applicable cutoff for both APRI and FIB-4.

Separately, a threshold of $>0.64$ for APRI score was $72 \%$ sensitive and $83 \%$ specific in the diagnosis of this group of 
patients, with PPV 88\% and NPV 63\%, indicating that APRI score was a good tool for ruling-in patients with significant fibrosis. The FIB-4 score with a cutoff value of 1.46 was $81.5 \%$ sensitive and $79 \%$ specific in the diagnosis of $\mathrm{F} 3 / \mathrm{F} 4$ patients, but was less effective than APRI at ruling-in these patients (PPV $85.5 \%)$. However, when the 2 scores were considered together, the combination of both APRI and FIB- 4 with threshold values of 0.64 and 1.46, respectively, led to a more robust model that could predict patients who had significant fibrosis with a PPV of $93 \%$.

Several optimal APRI cutoff values for prediction of cirrhosis have been proposed in the literature [14]. In a systematic review, the lower recommended threshold cutoff value of 1 was $76 \%$ sensitive and $76 \%$ specific, with an overall AUROC of 0.76 . The PPV of this threshold was $32 \%$ while NPV was $94 \%$ [15]. The higher recommended threshold cutoff value of 2 was more specific (91\%), but less sensitive (49\%), with PPV 50\% and NPV 91\%, indicating its effectiveness in rulingout cirrhosis. A lower threshold of 0.5 and a higher one of 1.5 have been suggested in the literature as cutoff values for the identification of significant fibrosis. Se and Sp were $81 \%$ and $50 \%$ for the 0.5 cutoff, $35 \%$ and $91 \%$ for the 1.5 cutoff. PPV and NPV were calculated to be $59 \%$ and $75 \%$ for the 0.5 threshold, and $77 \%$ and $61 \%$ for the 1.5 threshold.

Regarding FIB-4 score, Vallet-Pichard et al evaluated its use in 847 patients with CHC in comparison to liver biopsy [16]. FIB- 4 with a threshold $<1.45$ had an NPV of $94.7 \%$ to exclude significant fibrosis, with a Se of $74.3 \%$, while a threshold higher than 3.25 had a PPV to confirm the existence of a significant fibrosis of $82.1 \%$, with a $\mathrm{Sp}$ of $98.2 \%$.

It is not unusual for threshold values to differ between published data. Differences in patient populations, including the prevalence of significant fibrosis, cirrhosis, and reference ranges used for AST and ALT levels, may explain these discrepancies [17]. Our data included a large number of patients with advanced fibrosis (63.5\%) or cirrhosis (46\%), quite a high proportion compared to literature data. The most important difference is that, in our study, biochemical markers were compared to TE-LS rather than liver biopsy, which remains the gold standard for liver fibrosis assessment.

In the era of economic crisis, and taking into account the high prices of antiviral drugs together with the need to simplify treatment in terms of HCV elimination, the question arises whether the use of biochemical scores such as APRI and FIB-4 could avoid TE or liver biopsy. Based on our findings, patients with advanced fibrosis could be defined relatively safely using a combination of APRI and FIB-4. But what about the noncirrhotic patients? In this context we also tried to find a best lower cutoff that could help in directly prescribing DAAs in $\mathrm{CHC}$ patients without the use of TE. Using a lower cutoff FIB4 score of 0.98 or less and an APRI of 0.3 or less we were able to distinguish F1/F2 patients who do not need TE. Taking together the 2 groups of patients (those with severe fibrosis and those with low fibrosis) we could obviate the need for TE assessment in $58.5 \%$ of referred patients, leaving TE to be used only in the "grey zone", which seems to contain less than 50\% of patients.

There are limitations to our study. The most important limitation was the use of a noninvasive technique such as TE for liver fibrosis assessment. Traditionally, liver biopsy has been considered as the "gold standard", but nowadays this invasive technique is no longer considered as the first-line method in routine daily practice in patients with viral hepatitis. TE has been evaluated by several studies and is considered as an effective noninvasive tool for liver fibrosis assessment $[6,8]$. Regarding the cutoff values we propose, they could be considered quite applicable in the group of the patients with severe fibrosis/cirrhosis; however, the limited number of patients in our cohort with F1/F2 make the cutoff we found in this group less relevant for low fibrosis. On the other hand, the retrospective nature of our study is the other main limitation. However, we used only well documented cases from our medical records who fulfilled our inclusion criteria, while the large number of participants allowed us to evaluate the scope of our study correctly.

In conclusion, our study demonstrated that the use of the marker we propose, the APRI-FIB-4-COMBO, namely the combination of APRI/FIB- 4 scores, with cutoff thresholds of 0.64 and 1.46 , respectively, could potentially be used to predict patients with significant fibrosis, while the use of a FIB-4 score

\section{Summary Box}

\section{What is already known:}

- Patients with cirrhosis or advanced fibrosis are of particular importance in chronic hepatitis $\mathrm{C}(\mathrm{CHC})$, as the choice of treatment regimen and the posttreatment prognosis depend on the stage of fibrosis

- Noninvasive methods for liver stiffness (LS) assessment include transient elastography (TE) and biochemical scoring systems such as APRI and FIB-4

- The diagnostic performance of APRI and FIB-4 scores compared to TE-LS in the detection of patients with cirrhosis or advanced fibrosis remains unclear

\section{What the new findings are:}

- Both scores predicted F4 patients adequately

- A FIB-4 score threshold of 1.63 could predict cirrhotic patients

- The combination of APRI/FIB-4 score thresholds of 0.64 and 1.46 , respectively, could potentially be used to predict patients with significant fibrosis, while score thresholds of 0.3 and 0.98 , respectively, detect patients with low fibrosis

- The effectiveness of APRI and FIB-4 scores is lower for resolving intermediate degrees of fibrosis

- In about $58 \%$ of $\mathrm{CHC}$ patients, TE-LS could possibly be avoided if the APRI-FIB-4-COMBO scoring system is used 
threshold of 1.63 could predict cirrhotic patients. Moreover, APRI-FIB-4-COMBO score thresholds of 0.3 and 0.98 respectively could potentially be used to define patients with low fibrosis. We believe that these markers could significantly reduce the need for TE-LS in the pretreatment evaluation of patients with $\mathrm{CHC}$. This would allow a more rapid treatment decision for patients with $\mathrm{CHC}$, which is of special significance in the era of a worldwide HCV elimination plan. Larger prospective studies are needed to confirm our findings.

\section{References}

1. Polaris Observatory HCV Collaborators. Global prevalence and genotype distribution of hepatitis C virus infection in 2015: a modelling study. Lancet Gastroenterol Hepatol 2017;2:161-176.

2. Stasi C, Silvestri C, Voller F, Cipriani F. The epidemiological changes of HCV and HBV infections in the era of new antiviral therapies and the anti-HBV vaccine. J Infect Public Health 2016;9:389-395.

3. European Association for the Study of the Liver. EASL Recommendations on Treatment of Hepatitis C 2018. J Hepatol 2018;69:461-511.

4. Afdhal NH, Bacon BR, Patel K, et al. Accuracy of Fibroscan, compared with histology, in analysis of liver fibrosis in patients with hepatitis B or C: a United States multicenter study. Clin Gastroenterol Hepatol 2015;13:772-779.e1-e3.

5. Chou R, Wasson N. Blood tests to diagnose fibrosis or cirrhosis in patients with chronic hepatitis C virus infection: a systematic review. Ann Intern Med 2013;158:807-820.

6. Castéra L, Vergniol J, Foucher J, et al. Prospective comparison of transient elastography, Fibrotest, APRI, and liver biopsy for the assessment of fibrosis in chronic hepatitis C. Gastroenterology 2005;128:343-350.

7. Castéra L, Sebastiani G, Le Bail B, de Lédinghen V, Couzigou P, Alberti A. Prospective comparison of two algorithms combining non-invasive methods for staging liver fibrosis in chronic hepatitis C. J Hepatol 2010;52:191-198.

8. Ziol M, Handra-Luca A, Kettaneh A, et al. Noninvasive assessment of liver fibrosis by measurement of stiffness in patients with chronic hepatitis C. Hepatology 2005;41:48-54.

9. Wai CT, Greenson JK, Fontana RJ, et al. A simple noninvasive index can predict both significant fibrosis and cirrhosis in patients with chronic hepatitis C. Hepatology 2003;38:518-526.

10. Sterling RK, Lissen E, Clumeck N, et al; APRICOT Clinical Investigators. Development of a simple noninvasive index to predict significant fibrosis in patients with HIV/HCV coinfection. Hepatology 2006;43:1317-1325.

11. Lin ZH, Xin YN, Dong QJ, et al. Performance of the aspartate aminotransferase-to-platelet ratio index for the staging of hepatitis C-related fibrosis: an updated meta-analysis. Hepatology 2011;53:726-736.

12. Kim BK, Kim DY, Park JY, et al. Validation of FIB-4 and comparison with other simple noninvasive indices for predicting liver fibrosis and cirrhosis in hepatitis B virus-infected patients. Liver Int 2010;30:546-553.

13. European Association for Study of the Liver; Asociacion Latinoamericana para el Estudio del Higado. EASL-ALEH Clinical Practice Guidelines: Non-invasive tests for evaluation of liver disease severity and prognosis. J Hepatol 2015;63:237-264.

14. Houot M, Ngo Y, Munteanu M, Marque S, Poynard T. Systematic review with meta-analysis: direct comparisons of biomarkers for the diagnosis of fibrosis in chronic hepatitis $\mathrm{C}$ and B. Aliment Pharmacol Ther 2016;43:16-29.

15. Shaheen AA, Myers RP. Diagnostic accuracy of the aspartate aminotransferase-to-platelet ratio index for the prediction of hepatitis C-related fibrosis: a systematic review. Hepatology 2007;46:912-921.

16. Vallet-Pichard A, Mallet V, Nalpas B, et al. FIB-4: an inexpensive and accurate marker of fibrosis in HCV infection. Comparison with liver biopsy and fibrotest. Hepatology 2007;46:32-36.

17. Wong S, Huynh D, Zhang F, Nguyen NQ. Use of aspartate aminotransferase to platelet ratio to reduce the need for FibroScan in the evaluation of liver fibrosis. World J Hepatol 2017;9:791-796. 